\title{
Isotropization of Bianchi-Type Cosmological Solutions in Brans-Dicke Theory
}

\author{
P. Chauvet* \\ Departamento de Física, \\ Universidad Autónoma Metropolitana-Iztapalapa \\ P. O. Box. 55-534, México D. F. \\ C. P. 09340 MEXICO \\ Fax 52-5-724 4611 \\ J.L.Cervantes-Cota ${ }^{\dagger}$ \\ University of Konstanz, p.o.box: 5560, M 678 \\ D-78434 Konstanz \\ Fax 49-7531-88 3888
}

PACS number: 04.20.Jb,

gr-qc/9502015

\begin{abstract}
The cosmic, general analitic solutions of the Brans-Dicke Theory for the flat space of homogeneous and isotropic models containing perfect, barotropic, fluids are seen to belong to a wider class of solutions -which includes cosmological models with the open and the closed spaces of the Friedmann-Robertson-Walker metric, as well as solutions for models with homogeneous but anisotropic spaces corresponding to the BianchiType metric clasification- when all these solutions are expressed in terms of reduced variables. The existence of such a class lies in the fact that the scalar field, $\phi$, times a function of the mean scale factor or "volume element", $a^{3}=a_{1} a_{2} a_{3}$, which depends on time and on the barotropic index of the equation of state used, can be written as a function of a "cosmic time" reduced in terms of another function of the mean scale factor depending itself again on the barotropic index but independent of the metrics here employed. This reduction procedure permites one to analyze if explicitly given anisotropic cosmological solutions "isotropize" in the course of their time evolution. For if so can happen, it could be claimed that there exists a subclass of solutions that is stable under anisotropic perturbations.
\end{abstract}

*e-mail:pcha@xanum.uam.mx

†e-mail: jorge@spock.physik.uni-konstanz.de 


\section{INTRODUCTION}

The first authors to realize the possibility of giving a reason for the viability of the "cosmological principle" without the necessity of imposing highly special initial conditions before the "inflationary programme" was developed, were Hoyle and Narlikar [1]. However, their explanation came also before the cosmic microwave background (CMB) radiation was discovered, and nowadays, most investigators believe that the steady state theory is untenable from the observations. Other investigators within Einstein's general relativity theory (EGR), notably Misner [2], tried to demonstrate - unsuccesfully- that the large scale structure of the Universe, in particular its isotropy, could be attributed to the nature of the matter processes, such as dissipation, that took place at a very early stage of development of the Universe independent of its initial conditions (chaotic cosmology), that is, that the Universe lost memory of any initially imposed anisotropy or inhomogeneity (Barrow and Matzner [3], Doroshkevich et. al. [4], Misner [5], Rees [6], Zel'dovich and Starobinsky [7]). More to the point, within theoretical cosmology in general and in the context of EGR in particular, one is still looking for a satisfactory explanation to the following observational facts: The formation of galaxies and cluster of galaxies which means that the universe is not homogeneous locally, and on the other hand the CMB radiation that seems to be very nearly isotropic on account of its Planck spectrum and the lack of structure in its intensity, from which it has been concluded that the large-scale structure of the actual Universe must be homogeneous and isotropic. The standard big bang model, thought to give the most accurate description of the Universe, has the peculiarity that it appears to need a very special set of initial conditions to be viable. This state of affairs have produced several studies in different but related directions to obtain reasonable explanations to this conundrum, like the "inflationary programme" which nowadays is a popular approach, nevertheless not without some drawbacks, to solve also some other problems in cosmology (for a present review see Olive [8]). A most important reason why cosmological models that predict inflation in the early universe are interesting is the hope they will explain the observed state of the universe without appeal to highly special initial conditions. Even so, most inflationary cosmological models have assumed Friedmann-Robertson-Walker (FRW) symmetry from the outset. The horizon size in the FRW models suggests the possibility that physical interactions could have homogenized and isotropized the universe, and therefore that its present state could have evolved from more general initial conditions. Yet, it is not established if general cosmological models with non FRW initial conditions that actually enter, and gracefully exit, an inflationary phase initial homogeneity and anisotropy will be smoothed out since gravitational interactions tend to enhance inhomogeneities instead of smoothing them out, see Wald [9].

On experimental grounds the degree of anisotropy of our Universe is somewhat 
bounded by the current measurements of the CMB anisotropy made by COBE [10]. Nevertheless, present observations are still far from elucidating all the properties that an actual model of the universe should have. Even the degree of anisotropy of the primeval radiation or the counts of radio sourcess or galaxies in the various directions in the sky involve some uncertainties and, agreement between the observed chemical composition and the predictions of the Friedmann models merely signifies that the time rate of change of the volume occupied by matter and the rate of expansion of a Friedmann model concide. On the other hand, astronomical observations seem to imply that isotropization must occur at a rather early epoch, maybe even for $z>>10^{9}$. It is feasible that for particular anisotropic models this could be achieved for the instantaneous values of the anisotropy parameters. But, the case is that there are observational properties that depend on the degree of anisotropy over an extended period of time, again like the CMB isotropy.

In EGR, without the aid of a cosmological constant or inflation, Collins and Hawking [11] examined the question in terms of an "initial conditions" analysis. They obtained that the set of spatially homogeneous cosmological models approaching isotropy in the limit of infinite times is of zero measure in the space of all spatially homogeneous models, which in turn implies that the isotropy of the models is unstable to homogeneous and anisotropic perturbations. Yet, other authors define the concept of isotropization in a different way (see Novikov [12], Mac Callum [13] and Zel'dovich and Novikov [14]). Therefore, in the literature concerned with the mathematical analysis of anisotropic models the term isotropization is often mentioned but its precise definition is author dependent. For the Bianchi-Type models it is claimed that a positive cosmological constant provides an effective mean of isotropizing homogeneous universes (Wald [9]). However, in this context Barrow [15] has shown that contrary to previous expectations, perfect fluid cosmologies need not approach isotropy and homogeneity as $t \rightarrow \infty$.

Following the general line of thought on this subject put forward by Zel'dovich and Novikov, one declares that homogeneous cosmological models that isotropize are those that "approach to a Friedmann model in the course of time as the universe expands", which means that "its geometric and dynamic parameters as well as those concerning the distribution and motion of matter and radiation are nearly the corresponding quantities in a Friedmann model". Accordingly, one assumes that the idea conveyed by isotropization is the property that an arbitrary solution possesses to model our Universe which permits it to evolve from an initial, general state, into a state that is presently isotropic on a "large scale" and is, therefore, well described by a Friedmann solution. We reduce the general scope of the problem by assuming initial homogeneity, limiting ourselves to test the isotropization properties of certain specific non inflationary solutions in the Jordan-Brans-Dicke (JBD) cosmological theory. So, if at its outset 
the universe was not in isotropic expansion, the above ideas imply that one can examine, on a first approximation, the properties of homogeneous but anisotropic models assumed to describe correctly the early stages of its expansion. Of these, only those Bianchi-Type models whose group type comprise FRW models may isotropize: Types $\mathrm{I}, \mathrm{V}, \mathrm{VII}_{0}, \mathrm{VII}_{h}$ and IX.

In this paper the above concept of isotropization is dealt with in a direct, but admittedly limited, way by qualifying and quantifying it through a "Raychaudhuri type" equation common to all Bianchi-Type models: Given an explicit solution, one can directly check if it may or may not approach to a Friedmann regime in the course of its cosmological time evolution, specifically, if the different anisotropic scale factors of a Bianchi model in the various directions approach arbitrarily near to a unique, single function of time. By this procedure one can then answer the question, at least for some representative spatially homogeneous models of the Bianchi- Type (I, V, IX), of whether, and if so, how in the JBD cosmological theory a present large scale isotropy resulted from an initially anisotropic but homogeneous expanding universe.

\section{FRW FIELD EQUATIONS}

The JBD field equations for the FRW cosmology with a barotropic, perfect fluid, $p=\beta \rho,-1<\beta<1$ (the $\beta=\frac{1}{3}$, equation of state for incoherent radiation or ultrarelativistic matter is excluded) are

$$
\begin{gathered}
\rho a^{3(1+\beta)}=M_{\beta}, \quad M_{\beta}=\text { const. } \\
3(1-\beta) \frac{a^{\prime}}{a}=\left(\frac{\psi^{\prime}}{\psi}\right)-\frac{(1-3 \beta) m_{\beta} \eta+\eta_{0}}{\psi}, \quad m_{\beta}=\frac{8 \pi M_{\beta}}{3+2 \omega} .
\end{gathered}
$$

The dynamic equation is

$$
\left(\frac{\psi^{\prime \prime}}{\psi}\right)-\frac{\left[2(2-3 \beta)+3(1-\beta)^{2} \omega\right] m_{\beta}}{\psi}=\frac{-6(1-\beta) k}{a^{2(1-3 \beta)}} \quad, \quad k=0, \quad \pm 1
$$

and the constraint equation is

$$
\begin{aligned}
& \frac{3}{2(1-\beta)}\left(\frac{\psi^{\prime \prime}}{\psi}\right)-\frac{1}{(1-\beta)^{2}}\left(\frac{\psi^{\prime}}{\psi}\right)^{2}-\frac{(1-3 \beta)}{(1-\beta)^{2}}\left(\frac{(1-3 \beta) m_{\beta} \eta+\eta_{0}}{\psi}\right)\left(\frac{\psi^{\prime}}{\psi}\right) \\
+ & \frac{\left[2-3 \beta+\frac{3}{2}(1-\beta)^{2} \omega\right]}{(1-\beta)^{2}}\left(\frac{(1-3 \beta) m_{\beta} \eta+\eta_{0}}{\psi}\right)^{2}+\frac{3[2+\omega(1-\beta)(1+3 \beta)] m_{\beta}}{2(1-\beta) \psi} \\
= & 0,
\end{aligned}
$$


where $\psi \equiv \phi a^{3(1-\beta)}, \phi$ is the JBD scalar field, $a$ is the scale factor, $\omega$ the coupling parameter of the theory, $\eta$ the "cosmic time parameter", $\eta_{0}$ an integration constant, and ()$^{\prime}=\partial_{\eta}$, where $d t=a^{3 \beta} d \eta$ (for details see Chauvet and Pimentel [16], and references therein).

For $k=0$ equation (3) is directly integrated. One gets

$$
\psi=A \eta^{2}+B \eta+C
$$

where $\mathrm{A}, \mathrm{B}$ and $\mathrm{C}$ are constants such that

$$
A=\left[2-3 \beta+\frac{3}{2}(1-\beta)^{2} \omega\right] m_{\beta} .
$$

Substitution of (5) and (6) in the constraint equation hands out the following results. The constant $B$ is undetermined and so, up to $B, C$ also remains undetermined. Therefore, three different possible cosmic solutions to the FRW flat space $(k=0)$ exist distinguished by the sign of the determinant, $\Delta \equiv B^{2}-4 A C$, which itself depends on the relation between the equation of state, through $\beta$, and the coupling parameter $\omega$ in a rather complex way. The behavior of the scalar field $\phi$ implies, for each type of determinant, $\Delta>0, \Delta<0$ and $\Delta=0$, the possible existence of two branches: essentially, ones with $\phi$ an increasing function of time and the others with $\phi$ a decreasing function of time (the solutions are given explicitly and thoroughly discussed by Gurevich et.al. [17], Ruban and Finkelstein [18] and Morganstern [19]).

$\phi$ is obtained by the straightforward integration of

$$
\frac{\phi^{\prime}}{\phi}=\frac{(1-3 \beta) m_{\beta} \eta+\eta_{0}}{\psi},
$$

and the scale factor, $a$, found from it through the definition of $\psi$ :

$$
a^{3(1-\beta)}=\frac{A \eta^{2}+B \eta+C}{\phi} .
$$

Equation (4) does not involve the curvature constant $k$ explicitly and so, $\psi=$ $A \eta^{2}+B \eta+C$ is also a solution to actually both the open and closed space dynamic equation (3) provided that

$$
\phi a^{(1+3 \beta)}=\frac{2+(1-\beta)(1+3 \beta) \omega}{2(1+3 \beta) k} m_{\beta} .
$$

The same as in the flat space case, $A, B$ and $C$ are obtained from the constriction equation (四). For both, $k=+1$ and -1 , it is valid that

$$
A=\frac{-(1-3 \beta)^{2} m_{\beta}}{(1+3 \beta)} \text {, }
$$




$$
\begin{aligned}
& B=-2\left(\frac{1-3 \beta}{1+3 \beta}\right) \eta_{0}, \\
& C=-\frac{\eta_{0}^{2}}{(1+3 \beta) m_{\beta}} .
\end{aligned}
$$

Its determinant is then

$$
\Delta=B^{2}-4 A C=0 .
$$

The explicit solutions for these two models are

$$
\phi=\frac{-1}{(1+3 \beta) m_{\beta}}\left(\frac{[2+(1-\beta)(1+3 \beta) \omega] m_{\beta}^{2}}{-2 k}\right)^{\frac{3(1-\beta)}{2(1-3 \beta)}}\left[(1-3 \beta) m_{\beta} \eta+\eta_{0}\right]^{-\frac{1+3 \beta}{1-3 \beta}},
$$

and

$$
a=\left(\frac{-2 k}{[2+(1-\beta)(1+3 \beta) \omega] m_{\beta}^{2}}\right)^{\frac{1}{2(1-3 \beta)}}\left[(1-3 \beta) m_{\beta} \eta+\eta_{0}\right]^{\frac{1}{1-3 \beta}} .
$$

The solutions for the JBD flat space were previously obtained by several authors (Gurevich et. al. [17], Morganstern [19] and references therein, and in another context by Chauvet and Pimentel [16]).

Next, we present the anisotropic Bianchi field equations in the above variables in order to analyze later their asymptotic solutions.

\section{ANISOTROPIC FIELD EQUATIONS}

Three extra equations, and simple modifications to the FRW equations (11)-(丑) presented above, describe the Bianchi-Types I, V and IX examined in this paper. Equations (1) and (2) remain formally the same, while equation (3) gets its "curvature" term modified and is then written

$$
\left(\frac{\psi^{\prime \prime}}{\psi}\right)-\frac{\left[2(2-3 \beta)+3(1-\beta)^{2} \omega\right] m_{\beta}}{\psi}=(1-\beta) a^{6 \beta *} R_{j} .
$$

The constriction equation is a "Raychaudhuri type" equation so that the left hand side

of equation (田) remains unaltered, but instead of being equal to zero as in the FRW cosmology, it is in this case:

$$
\begin{gathered}
\frac{3}{2(1-\beta)}\left(\frac{\psi^{\prime \prime}}{\psi}\right)-\frac{1}{(1-\beta)^{2}}\left(\frac{\psi^{\prime}}{\psi}\right)^{2}-\frac{(1-3 \beta)}{(1-\beta)^{2}}\left(\frac{(1-3 \beta) m_{\beta} \eta+\eta_{0}}{\psi}\right)\left(\frac{\psi^{\prime}}{\psi}\right) \\
+\frac{\left[2-3 \beta+\frac{3}{2} \omega(1-\beta)^{2}\right]}{(1-\beta)^{2}}\left(\frac{(1-3 \beta) m_{\beta} \eta+\eta_{0}}{\psi}\right)^{2}+\frac{3[2+\omega(1-\beta)(1+3 \beta)] m_{\beta}}{2(1-\beta) \psi} \\
=-\left(H_{1}-H_{2}\right)^{2}-\left(H_{2}-H_{3}\right)^{2}-\left(H_{3}-H_{1}\right)^{2} \equiv \sigma(\eta) .
\end{gathered}
$$


$\sigma$ is, for short, the "shear". $\sigma=0$, is a necessary condition to obtain a FRW cosmology since it implies $H_{1}=H_{2}=H_{3}$ ( see Chauvet et.al. [20]). If the sum of the squared differences of the Hubble expansion rates tends to zero it would mean that anisotropic scale factors tend to a single function of time which is, presumably, the scale factor of a corresponding Friedmann model. However, in general not all Bianchi models contain a FRW space-time.

The three extra equations describe the dynamical evolution of the "anisotropic scale factors" $a_{1}, a_{2}$ and $a_{3}$ :

$$
\left(\psi H_{i}\right)^{\prime}=[1+(1-\beta) \omega] m_{\beta}+\psi a^{6 \beta *} R_{i j} . \quad \mathrm{i}=1,2,3
$$

From equations (14) through (16), and for the rest of this paper, we use the following notation and conventions: $a$ is presently the mean scale factor, $a^{3}=a_{1} a_{2} a_{3}$, the $H_{i}$ 's $i=1,2,3$ are the Hubble expansion rates, $H_{i}=a_{i}^{\prime} / a_{i},{ }^{*} R_{j}$ is the "spatial threecurvature" that belongs to a given Bianchi-Type model, ${ }^{*} R_{j}=\sum_{i=1}^{3}{ }^{*} R_{i j}$ is a column sum, and the ${ }^{*} R_{i j}$ are "partial curvature" terms pertaining to specific scale factor dynamic equations, in our case:

$$
\begin{array}{ccc} 
& V & I X \\
& & \\
& & \\
{ }^{*} R_{i j}= & 2 / a_{1}^{2} & {\left[a_{1}^{4}-a_{2}^{4}-a_{3}^{4}+2 a_{2}^{2} a_{3}^{2}\right] /\left(-2 a^{6}\right)} \\
0 & 2 / a_{1}^{2} & {\left[a_{2}^{4}-a_{3}^{4}-a_{1}^{4}+2 a_{1}^{2} a_{3}^{2}\right] /\left(-2 a^{6}\right)} \\
0 & 2 / a_{1}^{2} & {\left[a_{3}^{4}-a_{1}^{4}-a_{2}^{4}+2 a_{1}^{2} a_{2}^{2}\right] /\left(-2 a^{6}\right)}
\end{array}
$$

In the next section it is first shown that $\psi=A \eta^{2}+B \eta+C$ includes solutions for the above, purposely chosen homogeneous but anisotropic, cosmological models. It will be shown that the obtained $\psi$ class of solutions consist of two parts: the isotropic and the anisotropic one. Then, it will be clear that the latter approach to zero as the cosmic time parameter evolves, i.e., these solutions tend asymptotically to their corresponding isotropic group solutions, the FRW models.

\section{ANISOTROPIC SOLUTIONS AND THEIR ASYMPTOTIC BEHAVIOR}

We show next that $\psi=A \eta^{2}+B \eta+C$ is a solution for the, homogeneous but anisotropic, Bianchi-Type cosmological models.

\section{Bianchi- type I}


For this Bianchi type model, ${ }^{*} R_{I}=0$. It is direct to see by substituting

$$
\psi=A \eta^{2}+B \eta+C
$$

into equation (14), that $A$ has the same expresion as the one given by equation (6) (from now on we attach a subindex to the $A, B$ and $C$ to distinguish between the different Bianchi models):

$$
A_{I}=\left[2-3 \beta+\frac{3}{2}(1-\beta)^{2} \omega\right] m_{\beta}
$$

By direct substitution of the above results into equation (15) one finds that $B_{I}$ remains undetermined and may be put equal to any convenient, but arbitrary value, and that

$$
\begin{aligned}
& 3(1-\beta)^{2}(3+2 \omega) m_{\beta} C_{I}= \\
& \quad-\frac{(1-\beta)^{2}}{\omega^{3}}\left(h_{1}^{2}+h_{2}^{2}+h_{3}^{2}\right)-m_{\beta} \eta_{0}^{2} A_{I}+B_{I}{ }^{2}+(1-3 \beta) m_{\beta} \eta_{0} B_{I},
\end{aligned}
$$

where the $h_{i}$ 's are constants such that

$$
H_{i}=\frac{1}{3} \frac{a^{3^{\prime}}}{a^{3}}+\frac{h_{i}}{\psi},
$$

The non-vanishing constants $h_{i}$ 's determine the anisotropic character of the solutions. They obey the condition

$$
h_{1}+h_{2}+h_{3}=0 \text {. }
$$

By integration of equation (20), using equation (2) and equation (5), one finds explicitly $a=a(\eta)$ (first obtained by Ruban and Finkelstein [18], see also Chauvet and Guzmán [21] and Chauvet [22]).

For the Bianchi's in general, when equation (5) is substituted into the Raychaudhuri equation (15), one obtains the shear as a function of $\psi$ and the $h_{i}$ 's,

$$
\sigma(\eta)=-\frac{3\left(h_{1}^{2}+h_{2}^{2}+h_{3}^{2}\right)}{2 \psi^{2}}
$$

This term permits, beside the only one allowed $\Delta=0$ solution for the $k \neq 0$, FRW models, two other solutions with $\Delta \neq 0$ such that $\sigma \rightarrow 0$ as $\eta \rightarrow \infty$ (or $t \rightarrow \infty$ ). It is in this sense that these solutions may isotropize in the course of their time evolution (note a mathematical characteristic of the anisotropic solutions shown by the above results, i.e., the relation between the exponents $h_{i^{\prime} s}$ and the $B$ and $C$ coeficients).

\section{Bianchi- type V}


Equation (5) is a solution for this Bianchi model, with $A, B$ and $C$ equal to

$$
\begin{aligned}
& A_{V}=-\frac{(1-3 \beta)^{2} m_{\beta}}{(1+3 \beta)} \\
& B_{V}=-2\left(\frac{1-3 \beta}{1+3 \beta}\right) \eta_{0} .
\end{aligned}
$$

$A_{V}$ and $B_{V}$ are equal to the ones obtained for the isotropic, $k= \pm 1$ cases, but

$$
m_{\beta}(1+3 \beta) C_{V}=-\frac{(1+3 \beta)^{2}\left(h_{1}^{2}+h_{2}^{2}+h_{3}^{2}\right)}{18 \beta+\omega(1+3 \beta)^{2}}-\eta_{0}^{2}
$$

So that,

$$
\phi=\left[\frac{[2+(1-\beta)(1+3 \beta) \omega] m_{\beta}}{-2(1+3 \beta)}\right]^{\frac{3(1-\beta)}{2(1-3 \beta)}}\left[A_{V} \eta^{2}+B_{V} \eta+C_{V}\right]^{-\frac{1+3 \beta}{2(1-3 \beta)}},
$$

and

$$
H_{1}=\frac{1}{3} \frac{a^{3^{\prime}}}{a^{3}}=\frac{a_{1}^{\prime}}{a_{1}}=-\frac{1}{(1+3 \beta)} \frac{(1-3 \beta) m_{\beta} \eta+\eta_{0}}{\left(A_{V} \eta^{2}+B_{V} \eta+C_{V}\right)} .
$$

The scale factors are

$$
a_{1}=\left[\frac{-2(1+3 \beta)}{[2+(1-\beta)(1+3 \beta) \omega] m_{\beta}}\right]^{\frac{1}{2(1-3 \beta)}}\left[A_{V} \eta^{2}+B_{V} \eta+C_{V}\right]^{\frac{1}{2(1-3 \beta)}}
$$

and

$$
a_{2}=a_{1} \exp \left[\frac{-2 h_{2}}{\sqrt{\Delta}} \operatorname{arctanh}\left[\frac{-2(1-3 \beta)\left[(1-3 \beta) m_{\beta} \eta+\eta_{0}\right]}{(1+3 \beta) \sqrt{\Delta}}\right]\right], \Delta>0
$$

or

$$
a_{2}=a_{1} \exp \left[\frac{2 h_{2}}{\sqrt{-\Delta}} \arctan \left[\frac{-2(1-3 \beta)\left[(1-3 \beta) m_{\beta} \eta+\eta_{0}\right]}{(1+3 \beta) \sqrt{-\Delta}}\right]\right], \Delta<0
$$

with

$$
a_{2} a_{3}=a_{1}^{2}
$$

These solutions are new. However, for $\Delta=0$,

$$
a_{3}=a_{2}=a_{1}
$$

is obtained. This last solution is clearly seen to be the one previously obtained for the isotropic, FRW model, with an open space $(k=-1)$. Again, the $\Delta=0$ is obtained only if $h_{1}=h_{2}=h_{3}=0$.

Independent of the value $\Delta$ might have, $h_{1}+h_{2}+h_{3}=0$ is always true. In the Type $\mathrm{V}$ models with $\Delta \neq 0$ one must have that $h_{2}=-h_{3}$ with $h_{1}=0$. For the latter case, truly anisotropic solutions are obtained with

$$
\Delta=B_{V}^{2}-4 A_{V} C_{V}=\frac{-8(1-3 \beta)^{2}}{18 \beta+(1+3 \beta)^{2} \omega} h_{2}^{2} .
$$


$C_{V}$, being proportional to the sum of the squares of the constants $h_{2}$ and $h_{3}$, carries the information concerning the nature of the anisotropic character of this Bianchi-Type model.

Since equation (22) holds for all the Type $\mathrm{V}$ models, the $\Delta \neq 0$ solutions could have had asymptotic behaviors to call them "nearly isotropic in appearance" if $\sigma \rightarrow 0$, when $\eta \rightarrow \infty$. In this regard one finds that: for the $\Delta<0$ solution $a_{2}$ tends to $a_{1} \exp \left(\pi h_{2} / \sqrt{-\Delta}\right)$ and $a_{3}$ tends to $a_{1} \exp \left(-\pi h_{2} / \sqrt{-\Delta}\right)$ so, when $\eta$ reaches the value $\left(100 \sqrt{-\Delta}-B_{V}\right) / 2 A_{V}$ these two scale factors differ from each other by one percent, and this solution is then "ninety nine percent near" the $\Delta=0$ solution, corresponding to the FRW cosmology for $k=-1$ (see equations (12) and (13)). On the other hand, for the $\Delta>0$ model the scale factors can never approach to a same, single, function of $\eta$. The reason for this is that $\eta$ is bounded, see equation (28). Nevertheless, it is significant that this last can be an inflationary solution.

\section{Bianchi type IX}

$\psi$ given by equation (5) is, likewise, a solution for this model with the $H_{i}$ 's given by equation (20). However, in this case the $h_{i}$ 's cannot be constants. Instead, the $h_{i}^{\prime} s=h_{i}(\eta)^{\prime} s$ are now new, and unknown, functions of $\eta$.

With equation (5) substituted into

$$
\left[H_{i} \psi\right]^{\prime}=[1+(1-\beta) \omega] m_{\beta}+a^{6 \beta} \psi^{*} R_{i_{I X}} \quad, \quad \mathrm{i}=1,2,3
$$

one must solve for

$$
h_{i}^{\prime}=a^{6 \beta} \psi^{*} R_{i I X}+\frac{2 A_{I X}-\left[2(2-3 \beta)+3(1-\beta)^{2} \omega\right] m_{\beta}}{3(1-\beta)} . \quad \mathrm{i}=1,2,3
$$

The sum of the above three equations,

$$
a^{6 \beta} \psi^{*} R_{i I X}=\frac{2 A_{I X}-\left[2(2-3 \beta)+3(1-\beta)^{2} \omega\right] m_{\beta}}{(1-\beta)}
$$

is, given explicitly in terms of $a_{1}, a_{2}$, and $a_{3}$,

$$
\frac{a_{1}^{4}+a_{2}^{4}+a_{3}^{4}-2\left(a_{1}^{2} a_{2}^{2}+a_{1}^{2} a_{3}^{2}+a_{2}^{2} a_{3}^{2}\right)}{2 a^{6(1-\beta)}}=\frac{\left[2(2-3 \beta)+3(1-\beta)^{2} \omega\right] m_{\beta}-2 A_{I X}}{(1-\beta) \psi},
$$

from which any chosen scale factor can be solved as function of the other two remaining ones.

On the other hand, equation (15) gives

$$
h_{1}^{2}+h_{2}^{2}+h_{3}^{2} \equiv \mathrm{K}^{2}=-\frac{\omega^{3}}{2(1-\beta)^{2}}\left[P \eta^{2}+Q \eta+S\right]
$$


where the $P, Q$ and $S$ constants, given in terms of $A_{I X}, B_{I X}$ and $C_{I X}$, stand for

$$
\begin{gathered}
P=X A_{I X}-\left[4 A_{I X}-Y\right](1-3 \beta)^{2} m_{\beta} \\
Q=X B_{I X}-\left[4 A_{I X} \eta_{0}-2 Y m_{\beta} \eta_{0}+2(1-3 \beta) B_{I X}\right](1-3 \beta) m_{\beta}
\end{gathered}
$$

and

$$
S=X C_{I X}-\left[2 \Delta+2(1-3 \beta) m_{\beta} \eta_{0} B_{I X}-Y m_{\beta}^{2} \eta_{0}^{2}\right]
$$

where

$$
X=3(1+3 \beta)(1-\beta)^{2} \omega m_{\beta}+6(1-\beta) m_{\beta}-2(1+3 \beta) A_{I X}
$$

and

$$
Y=2(2-3 \beta)+3(1-\beta)^{2} \omega
$$

The isotropic model solution that belongs to this Bianchi-Type model is obtained when $\Delta=0$, where one has that $h_{1}(\eta)=h_{2}(\eta)=h_{3}(\eta)=0$ (see section 2$)$.

Under any circumstance the functions $h_{i}$ 's, which must still obey the condition $h_{1}+h_{2}+h_{3}=0$, determine the anisotropic character of the solutions.

The $h_{i}$ 's can be given as

$$
\begin{gathered}
h_{1}=-\left[\frac{\kappa^{2}+4 \kappa+1}{3\left(\kappa^{2}+\kappa+1\right)}\right] \mathrm{K}, \\
h_{2}=\left[\frac{-\kappa^{2}+2 \kappa+2}{3\left(\kappa^{2}+\kappa+1\right)}\right] \mathrm{K},
\end{gathered}
$$

and

$$
h_{3}=\left[\frac{2 \kappa^{2}+2 \kappa-1}{3\left(\kappa^{2}+\kappa+1\right)}\right] \mathrm{K},
$$

where now $\kappa$ is another, new and yet unknown, function of $\eta$ : Unfortunatly, for $\Delta \neq 0$ we were not able to obtain the explicit functional dependence of $\kappa=\kappa(\eta)$. Even so, an asymptotic isotropic behavior, similar to other models, for the present solutions is also expected based on the strength of equations (22) and (37).

\section{DISCUSSION AND CONCLUSIONS}

The JBD cosmological equations for perfect fluids with barotropic equations of state is seen capable of beeing displayed, through the use of reduced variables, in a way which first permits one to obtain non trivial, significant solutions with little effort and next, but more important, to express them in terms of the single function $\psi=$ 
$A \eta^{2}+B \eta+C$. The fact is that the aforementioned solutions belong to a class which embraces Bianchi-Type models some of which, in turn, comprise the FRW isotropy groups. Moreover, stated explicitly this class contains the general (analytic) matter solutions for the Bianchi-Type I model as well as solutions for the other two BianchiTypes examined in this paper, which in turn include special ones, a subclass, that tend aymptotically, as $\eta \rightarrow \infty$, to corresponding FRW solutions. The reason for the existence of this set is that the functional form of the product of the scalar field, $\phi$, times a power of the mean scale factor, $a^{3}=a_{1} a_{2} a_{3}$, as a function of the time parameter $\eta$ is a solution to the equations used in this work independent of the metrics that give rise to any possible present anisotropy for Bianchi-Types I, V and IX models, and it has the FRW form. In other words, for a perfect fluid with a barotropic equation of state, we have shown that there exists a class of solutions for the Bianchi I, V and IX types that contain their corresponding FRW models. The Type V solutions are new, as well as those for the Type IX, but in the latter case because of the complexity of the curvature terms it is only possible to give the explicit form, in terms of $\eta$, of the scale factors $a_{i}$ 's up to the single, unknown, function $\kappa=\kappa(\eta)$. Nevertheless if $\eta \rightarrow \infty$, an asymptotic isotropic behavior for the $\Delta \neq 0$ solution should be expected in view of equations (22) and (37). Moreover, there are also other solutions obtained from $\psi$, through equation (5), which describe other Bianchi-Type models, see Chauvet and Guzmán [21]. All of the above are salient and remarkable properties of the class of solutions that we have found. Even more, the $\psi$ solutions also include the asymptotic solutions, these are the Type I models $(\Delta=0,>0$ and $<0)$, for all Bianchi-Types near the initial singularity, when the spatial curvature terms can be neglected. They are in this sense -up to the matter terms- comparable to the Kasner vacuum solution of EGR.

At this point we want to mention that, in JBD, the separate cosmological models are to be distinguished between themselves in a first instance, through the different values that the constants $A, B$ and $C$ obtain. Also significant is the fact that $B$ and $C$ carry the physical information on the nature of the presence, or even the absence, of the anisotropy that any given models may have. Remind that these constants also determine the value of the discriminant $\Delta$ in terms of the physical parameters $\beta$ and $\omega$. We stress the fact that solutions for the Bianchi models corresponding to $\Delta=$ 0 that possess an isotropy group, recover the FRW solutions: Type I goes into the corresponding flat FRW one, Type $\mathrm{V}$ into the open FRW one and the Type IX into the closed FRW one. Meanwhile, for the anisotropic solutions with $\Delta \neq 0$ the models isotropize in the course of their time evolution by tending in the Type I case to its corresponding FRW model, while for Types V and IX their evolution is toward the $\Delta=0$ solution which is the only one available to describe a non-flat FRW cosmology in the JBD context. 
The present observational evidence points to a high degree of isotropy of the, assumed relic, CMB radiation and, if so, it is a decisive argument in favour of the, nowadays, large scale homogeneous and isotropic expansion of the Universe. Then, if the initial stages of the expansion had a homogeneous but anisotropic behavior one could follow, within the JDB, how an actual nearly isotropic expansion can come about.

Acknowledgment This work was supported by CONACYT grant 400200-5-3672E. 


\section{References}

[1] F. Hoyle and J.V. Narlikar, Proc. R. Soc. A 273, 1 (1963).

[2] C.W. Misner, Nature 214, 30, (1967).

[3] J.D. Barrow and R.A. Matzner, Mon. Not. R. Astron. Soc. 181, 719, (1977).

[4] A.G. Doroshkevich, Y.B. Zel'dovich and I.D. Novikov, Sov. Phys. JETP 26, 408, (1968).

[5] C.W. Misner, Astrophys. J. 151, 431, (1968).

[6] M.J. Rees, Phys. Rev. Lett. 28, 1969, (1972).

[7] Y.B. Zel'dovich and A.A. Starobinsky, Sov. Phys. JETP 34, 1159, (1972).

[8] K.A. Olive, Phys. Reports 190, 307, (1990).

[9] R.M. Wald, Phys. Rev. D 28, 2118, (1983).

[10] G.F. Smoot et al, Astrophys. J. 396, L1, (1992); C.L. Bennett et al, Astrophys. J. 436, 423, (1994).

[11] C.B. Collins and S.W. Hawking, Ap. J. 180, 317, (1973).

[12] S.P. Novikov, Sov. Phys.: JETP, 35, 1031, (1972).

[13] M.A.H. Mac Callum, General Relativity, an Einstein Centenary Survey, S. W. Hawking and W. Israel, Ed, Cambridge Univ. Press, 533, (1979).

[14] Y.B. Zel'dovich and I.D. Novikov, Relativistic Astrophysics Vol. 2, University of Chicago Press, (1983).

[15] J.B. Barrow, Phys. Lett. B 187, 12, (1987).

[16] P. Chauvet and O. Pimentel, Gen. Rel. Grav. 24, 243, (1992).

[17] L.E. Gurevich, A.M. Finkelstein and V.A. Ruban, Astrophys. Space Sci. 22, 231, (1973).

[18] V.A. Ruban and A.M. Finkelstein, Gen. Rel. Grav. 6, 601, (1975).

[19] R.E. Morganstern, Phys. Rev. D4, 278, (1971). 
[20] P. Chauvet, J. Cervantes-Cota and H.N. Núñez-Yépez, Proceedings of the 7 th Latin American Symposium on General Relativity and Gravitation, SILARG VII, World Scientific, 487, (1991).

[21] P. Chauvet and E. Guzmán, Astrophys. Space Sci 126, 133, (1986).

[22] P. Chauvet, Astrophys. Space Sci 90, 51, (1983). 